\title{
Antibiotic treatment duration and prevention of complications in neonatal Staphylococcus aureus bacteraemia
}

\author{
S. Kempley ${ }^{a, *}$, O. Kapellou ${ }^{b}$, A. McWilliams ${ }^{a}$, J. Banerjee ${ }^{b}$, A. McCorqodale ${ }^{c}$, \\ M. Millar ${ }^{c}$ \\ a Barts and the London School of Medicine and Dentistry, Queen Mary University of London, UK \\ ${ }^{\mathrm{b}}$ Neonatal Unit, Homerton University NHS Foundation Trust, London, UK \\ c Barts Health NHS Trust, London, UK
}

\section{A R T I C L E I N F O}

\section{Article history:}

Received 23 December 2014

Accepted 21 July 2015

Available online $x x x$

\section{Keywords:}

Antibiotics

Complications

Infant newborn

Septicaemia

Staphylococcus aureus

\author{
S U M M A R Y
}

Background: In adults with Staphylococcus aureus bacteraemia, short duration of effective antibiotic treatment is associated with increased risk of complications and recurrence. The optimum duration of treatment for neonates is unknown and practice varies widely.

Aim: To relate the duration of treatment of neonatal S. aureus bacteraemia to prevention of complications and recurrence.

Methods: Retrospective cohort study of confirmed S. aureus bacteraemia occurring over a 10 year period in two large tertiary neonatal units. Neonatal patients developing confirmed S. aureus bacteraemia between birth and discharge from the neonatal unit were identified from microbiology department records. Clinical details obtained from case notes included demographics, duration of antibiotics and clinical outcomes. Recurrence was determined from laboratory and clinical records. Adverse outcomes were related to duration of antibiotic therapy.

Findings: A total of 90 infants had S. aureus bacteraemia, of which six were meticillinresistant S. aureus (7\%). Median gestation was 27 weeks (range: 23-41), birth weight $846 \mathrm{~g}(434-3840)$ and postnatal age 16 days (0-116). Adverse outcomes were found in $44 \%$, with death in $8 \%$. Median duration of appropriate antibiotics was 19 days (range: 0-54). There were no cases of recurrent bacteraemia after finishing antibiotics. There was no relationship between antibiotic duration and complications.

Conclusion: Neonatal S. aureus bacteraemia mainly affected preterm neonates and had a significant morbidity and mortality. Recurrent bacteraemia was rare, irrespective of treatment duration. For neonatal unit patients with S. aureus bacteraemia, antibiotic therapy for 14 days in uncomplicated cases may be sufficient to prevent recurrence, with longer treatment justified if there is inadequate source control.

(c) 2015 The Healthcare Infection Society. Published by Elsevier Ltd. All rights reserved.

\footnotetext{
* Corresponding author. Address: Centre for Paediatrics, Blizard Institute, Barts and the London School of Medicine and Dentistry, 4 Newark Street, London E1 2AT, UK. Tel.: +44 (0)20 7882 2615; fax: +44 (0)20 78822195.

E-mail address: s.t.kempley@qmul.ac.uk (S. Kempley).
} 


\section{Introduction}

Neonatal Staphylococcus aureus bacteraemia and sepsis remain important clinical problems. Recent surveillance reported S. aureus as second only to coagulase-negative staphylococcus as the cause of neonatal bacteraemia in the UK, and device-associated neonatal bacteraemia in the USA. ${ }^{1,2}$ Of these isolates, $8 \%$ in the UK and $33 \%$ in the USA were meticillinresistant (MRSA). A review of community-acquired neonatal sepsis in developing countries found $S$. aureus to be the most prevalent pathogen isolated. $^{3}$

In adults, S. aureus bacteraemia is frequently associated with metastatic disease, affecting heart valves, joints, kidneys, and central nervous system, contributing to morbidity and acting as a reservoir for recurrent disease. ${ }^{4}$ Recurrence is high if central lines are not removed. ${ }^{5}$ Some studies have demonstrated links between shorter treatment duration and recurrent disease, but others have not. ${ }^{5,6} \mathrm{~A}$ recent review highlighted the lack of clear evidence for most aspects of S. aureus bacteraemia management in adults, with only one randomized trial directly addressing antibiotic therapy duration. ${ }^{7,8}$ Their only clear conclusions were that infective foci must be identified and removed if possible, and that long-term antimicrobial therapy is required for persistent bacteraemia or an irremovable focus.

Recommended duration of therapy in adults depends on whether disease is complicated or uncomplicated. Complicated disease is defined by a positive follow-up blood culture at 48-96 h, community-acquired infection, persistent fever at $72 \mathrm{~h}$ or skin lesions suggesting systemic infection. Uncomplicated disease is indicated by catheter-associated infection with removal of the catheter, negative follow-up blood culture, defervescence within $72 \mathrm{~h}$, normal transoesophageal echocardiogram, no prosthetic material in joints or intravascular space, and no symptoms suggesting metastatic infection. ${ }^{\text {? }}$

Recent guidelines have been produced for treating MRSA bacteraemia in adults and children. Guidance from the UK recommended $\geq 14$ days of treatment for uncomplicated MRSA bacteraemia and longer for complicated disease, with no specific guidance for children. ${ }^{10}$ The Infectious Diseases Society of America recommended two weeks of treatment for uncomplicated MRSA bacteraemia and four to six weeks for complicated adult cases. In children they recommended intravenous vancomycin for two to six weeks with MRSA bacteraemia or proven endocarditis, with no specific recommendations for therapy duration in neonates. ${ }^{11}$

Prevention of recurrence is an important element within strategies to reduce the burden of hospital-acquired S. aureus infection in adults. There is little known about the burden of recurrent disease in neonatal unit populations or how it is influenced by antibiotic treatment duration. Several aspects of immune function in neonates explain their susceptibility to S. aureus infection. They rely on maternal IgG transferred across the placenta, which may be deficient if they are delivered prematurely. ${ }^{12}$ Neonates have reduced complement function, neutrophil chemotaxis and bacterial killing, suggesting that they may need longer therapy to prevent recurrence. ${ }^{13,14}$ This study aims to examine outcomes following $S$. aureus bacteraemia in neonates, relating the duration of antibiotic treatment to complications and recurrence.

\section{Methods}

\section{Procedures}

Laboratory microbiology databases were used to identify all cases of S. aureus bacteraemia in admissions to two large neonatal units over a 10-year period (2001-2010). When positive $S$. aureus cultures were taken, all infants were inpatients in the neonatal unit of either the Homerton Hospital (48 cases) or the Royal London Hospital (42 cases) in East London, UK. These provide neonatal care for their local populations and also take external medical and surgical referrals as tertiary neonatal intensive care units. Neither unit had standard guidance on duration of therapy for S. aureus bacteraemia and clinicians made decisions on an individual basis in conjunction with medical microbiologists.

Bacteraemia was defined by the isolation of S. aureus from a blood culture, taken from a peripheral vein. The units practised standard aseptic procedures for blood culture collection with skin preparation using a $70 \%$ isopropyl alcohol swab. Blood cultures were processed in accredited laboratories using standard operating procedures and automated systems. During this study, S. aureus isolates were not routinely tested for toxins. Clinical sepsis was defined as bacteraemia treated with antibiotics with clinical signs or a raised C-reactive protein (CRP). The study received approval from the clinical governance committees of both institutions (Reference nos.: 614/ 670 Homerton, 081-11 Royal London).

Clinical details were extracted from medical records, to identify risk factors at the time of infection and outcomes. Prior specified adverse outcomes were death, bacterial endocarditis, osteomyelitis, renal abscess, meningitis, brain abscess, pneumatocoele, duration of oxygen requirement, and recurrent $S$. aureus infection either during admission or during paediatric admission in the first year of life. Other nonspecified complications and adverse outcomes were recorded.

Appropriate antibiotics were defined as those to which the organism was identified as susceptible by standard methods (British Society for Antimicrobial Chemotherapy, Birmingham, UK). For orally absorbable drugs such as flucloxacillin, duration of appropriate antibiotics was the total of intravenous and oral.

Recurrence was defined as any clinical illness with isolation of S. aureus after stopping appropriate antibiotic therapy, recorded in the hospital or laboratory record during the first year of life, including both bacteraemia and localized disease. Breakthrough disease was defined as a positive S. aureus culture during the primary antibiotic course. Classical S. aureus disease was defined as abscess, osteomyelitis, impetigo, or scalded skin syndrome. Complicated disease was defined as $S$. aureus bacteraemia with a central line or implanted device which was not removed, or a focus which was hard for antibiotics to penetrate, such as a deep-seated abscess, or metastatic sites of infection. For three patients only, there was a protected focus (abscess or endocarditis), which could have arisen after the infection, or may have been present in a cryptic state before diagnosis. These cases were recorded as having an adverse outcome and complicated disease. 


\section{Statistical analysis}

Statistical analysis was performed to determine effects of appropriate antibiotic therapy duration on outcomes and recurrent infection. Patients who died while still receiving antibiotics were excluded from this analysis, as their treatment course was curtailed by death. Patients were divided into three groups according to antibiotic duration: <14 days, 14-27 days and $>27$ days. As several variables showed a skewed distribution, comparisons between groups were made using Kruskall-Wallis test for continuous variables and chi-square or Fisher's exact tests for categorical variables. Logistic regression was performed with adverse outcome as the dependent variable, and independent variables of birth weight, gender, meticillin-susceptibility, maximum CRP, antibiotic duration, and presence of complicated disease. All tests were performed using IBM SPSS Statistics v21.

\section{Results}

A total of 90 infants with $S$. aureus bacteraemia were identified, of which six (7\%) were MRSA. There was a preponderance of low gestation and birth weight (Table I), with median gestation of 27 weeks and median birth weight of $846 \mathrm{~g}$. Although median postnatal age was 16 days, peak incidence was in the second week of life (Figure 1).

An underlying diagnosis classically associated with $S$. aureus infection was reported in 10 cases $(11 \%)$; these were cellulitis or abscess (8), bone or joint infection (1) and line infection (1). In nine cases $(10 \%)$ there was pneumonia, which may be associated with S. aureus. Other diagnoses not normally associated with $S$. aureus included necrotizing enterocolitis and abdominal surgery (9), and congenital abnormality (5). Complicated S. aureus infection was found in $17(19 \%)$.

Adverse outcomes were found in 40 cases $(44 \%)$, which included recurrent or breakthrough $S$. aureus infection (4), death (7), osteomyelitis (2), limb shortening (1), meningitis (4), bacterial endocarditis (1), pneumatocoeles (1), surgical drainage of abscesses (2), necrotizing enterocolitis after the initial illness (6), and increased duration of oxygen requirement (16). Some had more than one adverse outcome.

\section{Recurrent infection}

There were no cases of recurrent S. aureus bacteraemia after completing the primary antibiotic course. Two infants had non-bacteraemic recurrent S. aureus infection; one with a respiratory infection requiring reintubation in the neonatal unit, the other with $S$. aureus otitis media during readmission to paediatric wards.

Two patients had breakthrough $S$. aureus infection during their primary antibiotic course. One had breakthrough S. aureus bacteraemia six days after starting antibiotics; this infant was colonized with S. aureus at birth and had S. aureus cultured from maternal expressed milk, so may have been reinfected. Another preterm infant with osteomyelitis had S. aureus cultured locally from abscesses eight days after starting treatment. There were no differences in clinical characteristics or antibiotic duration between those with recurrent or breakthrough disease and the remaining cohort.
Table I

Characteristics of the whole cohort of 90 infants with Staphylococcus aureus bacteraemia

\begin{tabular}{|c|c|}
\hline Characteristics & Values \\
\hline \multicolumn{2}{|l|}{ Demographics } \\
\hline Gestational age at birth (weeks) ${ }^{a}$ & $27(23-41)$ \\
\hline Birth weight $(\mathrm{g})^{\mathrm{a}}$ & $846(434-3840)$ \\
\hline Male gender & $49(54 \%)$ \\
\hline Inborn & $66(73 \%)$ \\
\hline \multicolumn{2}{|l|}{ Characteristics at time of positive culture } \\
\hline Postnatal age (days) ${ }^{a}$ & $16(0-116)$ \\
\hline Corrected GA at episode (weeks) ${ }^{a}$ & $31(23-53)$ \\
\hline Clinical sepsis & 88 (98\%) \\
\hline Classical clinical S. aureus disease & $10(11 \%)$ \\
\hline TPN within $48 \mathrm{~h}$ of culture & $54(60 \%)$ \\
\hline \multicolumn{2}{|l|}{ Enteral feeds during culture } \\
\hline Full & $30(33 \%)$ \\
\hline Partial & $49(54 \%)$ \\
\hline None & $10(10 \%)$ \\
\hline Central line when S. aureus isolated & $52(58 \%)$ \\
\hline MSSA & $84(93 \%)$ \\
\hline MRSA & $6(7 \%)$ \\
\hline \multicolumn{2}{|l|}{ Treatment } \\
\hline Total duration appropriate antibiotics (days) ${ }^{a}$ & $19(0-54)$ \\
\hline \multicolumn{2}{|c|}{ Duration of antibiotics } \\
\hline $0-13$ days & $16(18 \%)$ \\
\hline $14-27$ days & $52(58 \%)$ \\
\hline$\geq 28$ days & $21(23 \%)$ \\
\hline Vancomycin used & $41(46 \%)$ \\
\hline Central line removed during infection & $40(44 \%)$ \\
\hline $\begin{array}{l}\text { Complicated S. aureus infection (plastic } \\
\text { device remaining in situ or difficult- } \\
\text { to-penetrate site) }\end{array}$ & 17 (19\%) \\
\hline Antibiotics curtailed by death or discharge & $9(10 \%)$ \\
\hline \multicolumn{2}{|l|}{ Outcomes } \\
\hline Any adverse outcome & $40(44 \%)$ \\
\hline Death & $7(8 \%)$ \\
\hline Recurrent S. aureus infection & $4(4 \%)$ \\
\hline \multicolumn{2}{|l|}{ Recurrent S. aureus bacteraemia } \\
\hline $\begin{array}{l}\text { Breakthrough during primary } \\
\text { antibiotic course }\end{array}$ & 1 \\
\hline After completing antibiotics & 0 \\
\hline
\end{tabular}

GA, gestational age; TPN, total parenteral nutrition; MSSA, meticillinsusceptible S. aureus; MRSA, meticillin-resistant S. aureus.

${ }^{a}$ Median (range).

\section{Antibiotic duration and adverse outcomes}

Appropriate antibiotics were given for a median of 19 days (range: $0-54)$. Flucloxacillin without vancomycin was used in $51 \%$, vancomycin without flucloxacillin in $19 \%$, with both flucloxacillin and vancomycin used in $27 \%$. Aminoglycosides were used in $41 \%$. Antibiotics were given for a median duration of 21 days in complicated disease compared with 19 days in uncomplicated cases.

In nine patients the antibiotic course was curtailed by death or discharge to another unit; two had incomplete data on curtailment. Of 16 patients receiving antibiotics for less than two weeks, seven had their course curtailed, leaving 79 in whom the association between antibiotic duration and outcomes could be determined. Examining antibiotic duration by 

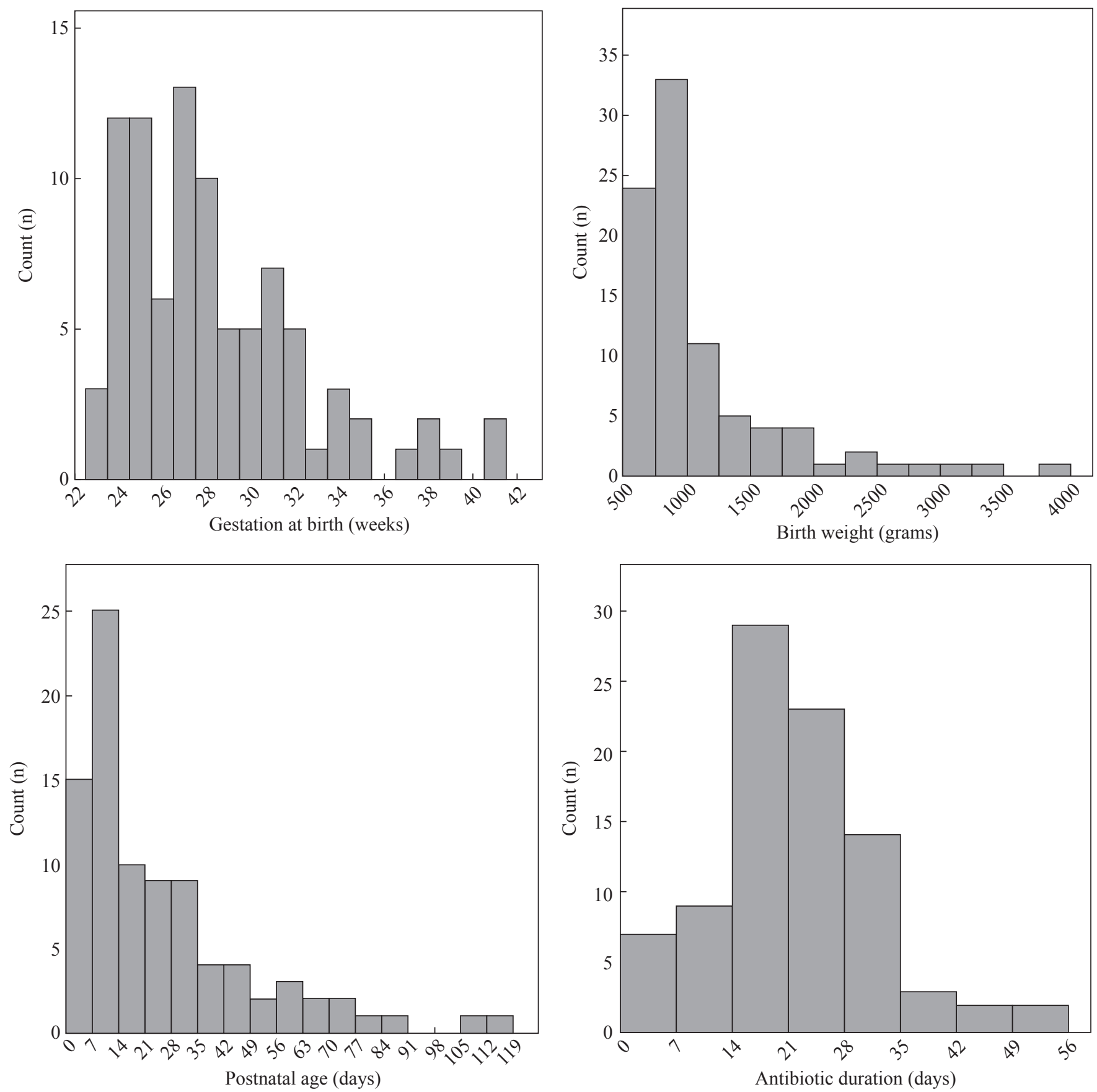

Figure 1. Gestation, birth weight, postnatal age distribution, and duration of antibiotic therapy. There was a preponderance of low gestation and low birth weight infants with peak incidence in the second week of life.

group ( $<14$ days, $14-27$ days, $>27$ days) there were no statistically significant differences in any parameter, but all those treated for $<14$ days had uncomplicated disease (Table II).

On univariate analysis, patients with adverse outcomes were more likely to be of low gestation or birth weight and to have complicated disease, and had a higher CRP (Table III). In stepwise logistic regression analysis, retained variables showed that the odds for adverse outcome increased with maximum CRP (odds ratio: 1.009; 95\% confidence interval: 1.000-1.018), were inversely related to birth weight $(0.998$; $0.996-0.999)$, and increased in the presence of complicated disease (16.97; 2.55-113.0).

\section{Deaths in S. aureus bacteraemia}

In three of the seven infants who died, staphylococcal sepsis was a stated cause of death. However, three others died soon after their positive $S$. aureus blood culture, suggesting that S. aureus infection contributed to death. Most who died were $<26$ weeks of gestation or $<1000 \mathrm{~g}$ birth weight. The only term infant who died had harlequin ichthyosis, with impaired cutaneous barrier function and a central line left in place. Both infants with MRSA who died received vancomycin. Median time from positive S. aureus blood culture to death was two days (range: 1-19). 
Table II

Characteristics and outcomes for the 79 patients whose antibiotic course was not curtailed by death or discharge

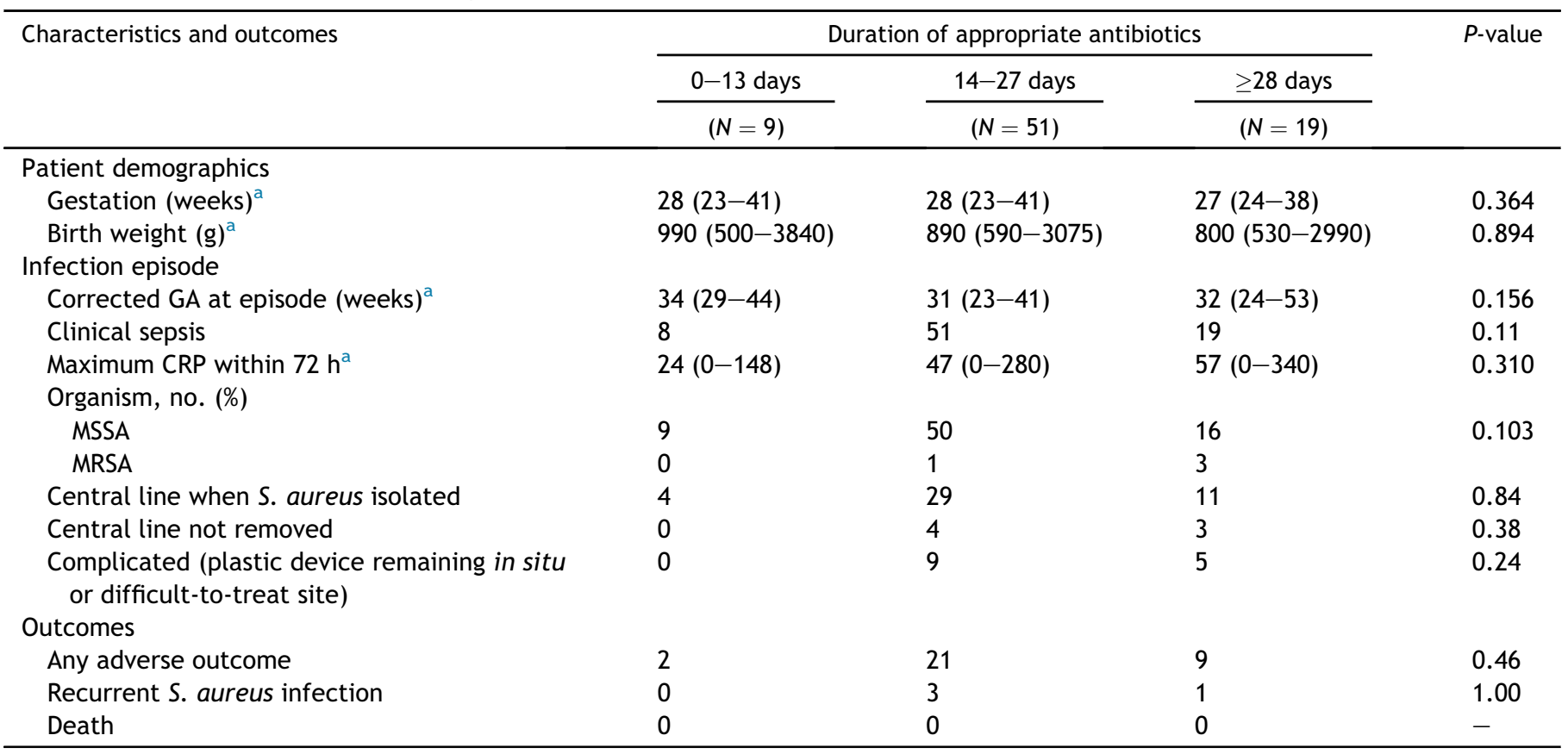

GA, gestational age; CRP, C-reactive protein; MSSA, meticillin-susceptible S. aureus; MRSA, meticillin-resistant S. aureus.

${ }^{a}$ Median (range).

\section{Discussion}

This study has described the clinical features of Staphylococcus aureus bacteraemia in a large cohort of infants admitted to neonatal units. The patients were predominantly preterm and many had associated conditions. There were high rates of complications and mortality, attributable to both S. aureus disease and underlying conditions. A predominance of disease at lower birth weight has been previously reported. ${ }^{2}$ Although $11 \%$ had a focus typical of $S$. aureus disease, the majority had bacteraemia without any focus.

Patients with adverse outcomes had lower gestation and birth weight, higher CRP and were more likely to have complicated disease, including retained central venous lines. This is consistent with research suggesting that central catheter retention is associated with adverse outcomes in neonates

Table III

Characteristics of patients with and without adverse outcomes

\begin{tabular}{|c|c|c|c|}
\hline Characteristics & No adverse outcome $(N=50)$ & Adverse outcome present $(N=40)$ & $P$-value \\
\hline \multicolumn{4}{|l|}{ Demographics } \\
\hline Gestation (weeks) $^{\mathrm{a}}$ & $28.5(23-41)$ & $25.5(23-37)$ & 0.001 \\
\hline Birth weight $(\mathrm{g})^{\mathrm{a}}$ & $980(500-3840)$ & $760(434-2990)$ & 0.006 \\
\hline Male sex & $26(52 \%)$ & $23(58 \%)$ & 0.60 \\
\hline \multicolumn{4}{|l|}{ Episode } \\
\hline Postnatal age (days) ${ }^{a}$ & $13(1-110)$ & $22.5(0-116)$ & 0.23 \\
\hline Corrected GA at episode (weeks) ${ }^{a}$ & $32.0(26-53)$ & $30.5(23-44)$ & 0.051 \\
\hline Maximum CRP within $72 \mathrm{~h}^{\mathrm{a}}$ & $32(0-193)$ & $63(0-340)$ & 0.023 \\
\hline Central line when S. aureus isolated & $31(62 \%)$ & $21(53 \%)$ & 0.44 \\
\hline Organism: MRSA & $1(2 \%)$ & $5(13 \%)$ & 0.09 \\
\hline \multicolumn{4}{|l|}{ Treatment } \\
\hline Total duration appropriate antibiotics (days) ${ }^{a}$ & $20(0-50)$ & $17(0-54)$ & 0.81 \\
\hline Vancomycin used & $19(38 \%)$ & $22(55 \%)$ & 0.084 \\
\hline Central line removed during infection & $28(56 \%)$ & $12(30 \%)$ & 0.026 \\
\hline $\begin{array}{l}\text { Complicated (plastic device remaining in situ } \\
\text { or difficult-to-treat site) }\end{array}$ & $3(6 \%)$ & $14(35 \%)$ & 0.001 \\
\hline
\end{tabular}

GA, gestational age; CRP, C-reactive protein; MRSA, meticillin-resistant S. aureus.

Analysis performed on all 90 patients.

${ }^{a}$ Median (range). 
with S. aureus or Gram-negative bacteraemia. ${ }^{15}$ In logistic regression, low birth weight, high CRP, and complicated disease carried an increased risk for adverse outcomes. These findings are limited by our study's retrospective design. Although patients with persisting disease markers were investigated for metastatic foci, this was decided individually by discussion between clinicians and microbiologists, with no fixed protocols for repeat blood cultures, further investigation, or antibiotic duration.

The guidelines for adults and children define uncomplicated disease by exclusion of endocarditis, with no metastatic sites or implanted prosthesis, and rapid disease control. ${ }^{11}$ This definition cannot be directly applied in neonates, in whom it is hard to exclude bacterial endocarditis. To retain the concept of difficult source control, complicated disease was defined as the presence of a central line or other foreign material which was not removed, a focus which was hard for antibiotics to penetrate, or metastatic sites of infection. Using this definition, complicated disease was not a risk factor for adverse outcome or recurrence, but all complicated cases had planned antibiotic courses of over two weeks. The favourable outcomes with less than two weeks of therapy may have been achieved because none had complicated disease.

There was no association between antibiotic duration and complications or recurrence. Only one infant was identified with bacterial endocarditis. In adults, Asgeirsson et al. found that in complicated $S$. aureus bacteraemia, duration of intravenous antibiotic therapy was 16 days in those experiencing a relapse and 30 days in those who did not. ${ }^{6}$ However, Walker et al. did not find an association between curtailed antibiotic therapy and recurrence. ${ }^{5}$ They found that recurrence was associated with keeping a central line in place and with glycopeptide treatment of meticillin-susceptible $S$. aureus.

No cases of recurrent bacteraemia were found after finishing antibiotics, and only one case of breakthrough bacteraemia occurred during treatment. Preterm infants in this study stayed in hospital for many weeks before discharge home, allowing detection of early recurrence. Although some patients were transferred in from other areas, $73 \%$ were inborn and would have been readmitted to the same hospital with recurrent disease. Although late recurrence rates could have been slightly underestimated, they were certainly very low. As endocarditis is a major factor in adult recurrent disease, this suggests that the newborn may be relatively well protected against $S$. aureus endocarditis. In vitro, shear stress reduces adherence of $S$. aureus to endothelial cells. ${ }^{16}$ Children and newborn infants have a higher cardiac output per unit body weight than adults, with the highest values in extremely preterm infants. ${ }^{17-19}$ This could produce greater shear forces at the endothelial interface and protect the normally vulnerable neonate against $S$. aureus endocarditis.

The proportion of our patients with MRSA was only $7 \%$, comparable with $8 \%$ found in another UK series, but lower than the $33 \%$ reported in a US series. ${ }^{1,2}$ A non-significant trend for more adverse outcomes with MRSA was found. Enhanced surveillance of MRSA bacteraemia in children reported a low proportion (1.9\%) with endocarditis. ${ }^{20}$ However, in a series of MRSA bacteraemia cases, Chuang et al. reported recurrent bacteraemia in seven out of $82(8.5 \%)$ infants; all were treated with $\leq 14$ days of vancomycin and four had catheter-related infection. ${ }^{21}$

Most infants who died were extremely preterm, suggesting that $S$. aureus bacteraemia may be particularly hazardous in this group. The approach to source control was adequate in those who died; only one infant with severe vascular access difficulties had a central line left in place. The median time from positive S. aureus blood culture to death was two days.

Our study is the largest series of neonatal S. aureus bacteraemia relating clinical outcomes to duration of antibiotic treatment. No link was found between duration of antibiotic therapy and adverse outcomes, but clinicians used longer courses of antibiotics for more complicated cases. Rates of endocarditis and recurrent bacteraemia were low. Given the problem of early mortality, the clinical priority is to implement strategies to prevent colonization and reduce early mortality. Removal of protected sources such as central lines may also be important to prevent recurrence. We suggest that in neonates with S. aureus bacteraemia it is reasonable to apply the adult recommendations for antibiotic therapy of 14 days in uncomplicated cases, and longer for cases with inadequate source control.

\section{Conflict of interest statement} None declared.

\section{Funding sources}

None.

\section{References}

1. Muller-Pebody B, Johnson AP, Heath PT, et al. Empirical treatment of neonatal sepsis: are the current guidelines adequate? Archs Dis Childh Fetal Neonatal Ed 2011;96:F4-F8.

2. Hocevar SN, Edwards JR, Horan TC, Morrell GC, Iwamoto M, Lessa FC. Device-associated infections among neonatal intensive care unit patients: incidence and associated pathogens reported to the National Healthcare Safety Network, 2006-2008. Infect Control Hosp Epidemiol 2012;33:1200-1206.

3. Downie L, Armiento R, Subhi R, Kelly J, Clifford V, Duke T. Community-acquired neonatal and infant sepsis in developing countries: efficacy of WHO's currently recommended antibiotics systematic review and meta-analysis. Archs Dis Childh 2013;98: $146-154$.

4. Mitchell DH, Howden BP. Diagnosis and management of Staphylococcus aureus bacteraemia. Intern Med J 2005;35(Suppl. 2): S17-S24.

5. Walker TM, Bowler ICJW, Bejon P. Risk factors for recurrence after Staphylococcus aureus bacteraemia. A retrospective matched case-control study. J Infect 2009;58:411-416.

6. Asgeirsson H, Kistjansson M, Kristinsson KG, Gudlaugsson 0 . Staphylococcus aureus bacteraemia - nationwide assessment of treatment adequacy and outcome. J Infect 2011;62:339-346.

7. Thwaites GE, Edgeworth JD, Gkrania-Klotsas E, et al. UK Clinical Infection Research Group. Clinical management of Staphylococcus aureus bacteraemia. Lancet Infect Dis 2011;11:208-222.

8. Rahal Jr JJ, Chan YK, Johnson G. Relationship of staphylococcal tolerance, teichoic acid antibody, and serum bactericidal activity to therapeutic outcome in Staphylococcus aureus bacteremia. Am J Med 1986;81:43-52.

9. Corey GR. Staphylococcus aureus bloodstream infections: definitions and treatment. Clin Infect Dis 2009;48(Suppl. 4):S254-S259.

10. Gould FK, Brindle R, Chadwick PR, et al. Guidelines (2008) for the prophylaxis and treatment of methicillin-resistant Staphylococcus aureus (MRSA) infections in the United Kingdom. J Antimicrob Chemother 2009;63:849-861.

11. Liu C, Bayer A, Cosgrove SE, et al. Clinical practice guidelines by the Infectious Diseases Society of America for the treatment of methicillin-resistant Staphylococcus aureus infections in adults 


\section{S. Kempley et al. / Journal of Hospital Infection xxx (2015) 1-7}

and children: executive summary. Clin Infect Dis 2011; 52:285-292.

12. Salimonu LS, Ladipo OA, Adeniran SO, Osukoya BO. Serum immunoglobulin levels in normal, premature and postmature newborns and their mothers. Int J Gynaecol Obstet 1978-1979;16:119-123.

13. Fleer A, Gerards LJ, Verhoef J. Host defence to bacterial infection in the neonate. J Hosp Infect 1988;11(Suppl. A):320-327.

14. Carr R. Neutrophil production and function in newborn infants. $\mathrm{Br}$ J Haematol 2000;110:18-28.

15. Benjamin Jr DK, Miller W, Garges H, et al. Bacteremia, central catheters, and neonates: when to pull the line. Pediatrics 2001;107:1272-1276.

16. Reddy K, Ross JM. Shear stress prevents fibronectin binding protein-mediated Staphylococcus aureus adhesion to resting endothelial cells. Infect Immun 2001;69:2472-2475.

17. de Simone G, Devereux RB, Daniels SR, et al. Stroke volume and cardiac output in normotensive children and adults. Assessment of relations with body size and impact of overweight. Circulation 1997; 95:1837-1843.

18. Groves AM, Chiesa G, Durighel G, et al. Functional cardiac MRI in preterm and term newborns. Archs Dis Childh Fetal Neonatal Ed 2010;96:F86-F91.

19. Sloot SC, de Waal KA, van der Lee JH, van Kaam AH. Central blood flow measurements in stable preterm infants after the transitional period. Archs Dis Childh Fetal Neonatal Ed 2010;95:F369-F372.

20. Johnson AP, Sharland M, Goodall CM, et al. Enhanced surveillance of methicillin-resistant Staphylococcus aureus (MRSA) bacteraemia in children in the UK and Ireland. Archs Dis Childh 2010;95:781-785.

21. Chuang YY, Huang YC, Lee CY, Lin TY, Lien R, Chou YH. Methicillin-resistant Staphylococcus aureus bacteraemia in neonatal intensive care units: an analysis of 90 episodes. Acta Paediatr 2004;93:786-790. 